\title{
Evapotranspiração e coeficiente de cultura da cenoura irrigada no agreste alagoano ${ }^{1}$
}

\author{
Julianna Catonio da Silva ${ }^{2 *}$, Cinara Bernardo da Silva², Daniella Pereira dos Santos ${ }^{3}$, \\ Márcio Aurélio Lins dos Santos ${ }^{2}$, Wanderson José de Oliveira ${ }^{3}$, Lígia Sampaio Reis ${ }^{4}$
}

\section{$10.1590 / 0034-737 X 201865040001$}

\begin{abstract}
RESUMO
O consumo hídrico de uma cultura é uma das principais informações necessárias para o manejo adequado da irrigação e um plano eficiente de uso da água. O objetivo deste trabalho foi encontrar o coeficiente de consumo de água para cultura da cenoura, comparando métodos de evapotranspiração de referência para a região agreste de Alagoas. $O$ experimento foi realizado no Campus Arapiraca da Universidade Federal de Alagoas, localizado na mesorregião Agreste do Estado. Foram utilizados cinco lisímetros de drenagem, com uma área de superfície de $0,07 \mathrm{~m}^{2}$, em que a determinação da evapotranspiração da cultura foi realizada diariamente, por meio de coletas de água dos drenos, obtidas diretamente dos lisímetros de drenagem. O coeficiente de cultivo foi calculado pela relação entre evapotranspiração da cultura e a de referência. Ao mesmo tempo, realizou-se uma análise comparativa dos modelos matemáticos de estimativa de evapotranspiração de referência utilizando os seguintes métodos: Penman-Monteith, Hargreaves-Samani, Radiação Solar, Blaney-Criddle e Priestley-Taylor. Os coeficientes de cultivo para a cultura da cenoura, recomendados para a região são: 0,46; 1,3; 1,3 e 1,03, para as fases I, II, III e IV, respectivamente, obtidos pelo método padrão. Os valores de evapotranspiração de referência obtidos pelo método Priestley-Taylor apresentaram os melhores desempenhos para os métodos estatísticos estudados e, na ausência das variáveis necessárias para se determinar a evapotranspiração de referência pelo método padrão, ele pode ser usado na região estudada.
\end{abstract}

Palavras-chave: lisímetro de drenagem; horticultura; consumo de água.

\section{ABSTRACT}

\section{Evapotranspiration and crop coefficient of the irrigated carrot on the alagoano agrest}

The hidric consumption of a crop is one of the main information required for proper irrigation management and to develop an efficient water usage plan. The objective of this work was to find a coefficient of water consumption for carrot crop, comparing methods of reference evapotranspiration for the agrest region of Alagoas. The experiment was carried out at the Arapiraca Campus of the Federal University of Alagoas, located in the Agrest mesoregion of the State of Alagoas. Were used five drainage lysimeters, with a surface area of $0.07 \mathrm{~m}^{2}$,on what the determination of crop evapotranspiration was performed daily, through the collection of water of the drains obtained directly from drainage lysimeters. The cultivation coefficient was calculated by the relation between crop evapotranspiration and reference evapotranspiration. At the same time, realized if a comparative analysis of the mathematical models of reference evapotranspiration estimation was carried out using the following methods: Penman-Monteith, Hargreaves-Samani, Solar Radiation Blaney-Criddle and Priestley-Taylor. The cultivation coefficient for carrot crop recommended for the region is $0.46 ; 1.3 ; 1.3$ and 1.03 for phases I, II, III and IV respectively by the standard method. The values of reference evapotranspiration obtained by the Priestley-Taylor method presented the best performances for the statistical methods studied, and in the absence of the necessary variables to determine the reference evapotranspiration by the standard method, it can be used for the studied region.

Keywords: drainage lisimetry; horticulture; water consumption.

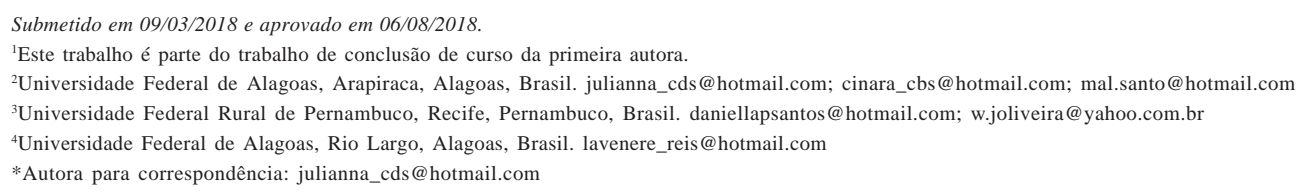




\section{INTRODUÇÃO}

A cenoura (Daucus carota L.) é uma das mais importantes olerícolas no cenário nacional, por seu elevado consumo. É uma hortaliça da família Apiaceae, do grupo das raízes tuberosas, cultivada em larga escala nas regiões Centro-Oeste, Sudeste, Nordeste e Sul do Brasil (Carvalho et al., 2017). No Agreste alagoano, a produção de cenoura encontra condições climáticas favoráveis ao seu desenvolvimento; porém, com a estação seca de longa duração, torna-se essencial o cultivo irrigado, com manejo racional da água (Lucena et al., 2016).

O sucesso da utilização da água para fins de irrigação depende do conhecimento preciso da demanda hídrica da cultura (Silva et al., 2012). Para isso, são necessárias informações sobre vários parâmetros, como a evapotranspiração da cultura (ETc), o coeficiente da cultura $(\mathrm{Kc})$ e evapotranspiração de referência (ETo).

Para a determinação do Kc são necessários a aplicação de algum método para estimar a ETo e o conhecimento da ETc para todas as fases de desenvolvimento (Miranda et al., 2016). No caso da cenoura, o seu Kc e consequentemente, a necessidade hídrica é um fator ainda pouco contemplado na literatura.

O método de Penman- Monteith, parametrizado pela FAO (Allen et al., 1998), é o método empírico recomendado para a estimativa da evapotranspiração de referência. Ele requer uma quantidade maior de elementos meteorológicos, que muitas vezes não estão disponíveis, ocasionando, assim, a busca de métodos de estimativa mais simples (Lucena et al., 2016) que empreguem menor número de variáveis, como os de Hargreaves-Samani, da Radiação Solar, de Blaney-Criddle e de Priestley-Taylor (Allen et al., 1998).

Os métodos de determinação da ETo normalmente estimam de forma satisfatória a evapotranspiração, nas condições de clima onde são desenvolvidos, mas, quando utilizados em condições diferentes podem ocasionar grandes erros e gerar grandes perdas de produção ou desperdício de recursos hídricos (Cavalcante Júnior, 2010). Assim sendo, o estudo local dessa variável é vital para o sucesso do plantio, como pode ser observado em muitos estudos que avaliam o desempenho dos diferentes métodos de estimativa da ETo (Araújo et al., 2011; Oliveira et al., 2015; Santos et al., 2016).

Para determinação da ETc, o lisímetro é uma forma de medi-la diretamente. Por definição, Pereira et al. (1997) estabeleceram que esse equipamento consiste em uma caixa impermeável, contendo um volume de solo e que permite conhecer com detalhe alguns termos do balanço hídrico do volume amostrado.

Sendo assim, objetiva-se com este estudo analisar vários métodos de estimativa da evapotranspiração de referência e encontrar os coeficientes de consumo hídricos da cultura da cenoura para a região agreste de Alagoas.

\section{MATERIAL E MÉTODOS}

O trabalho foi desenvolvido na unidade experimental do Grupo Irriga do Campus de Arapiraca, da Universidade Federal de Alagoas (UFAL), de março a julho de 2016, com dias juliano (J) variando de 91 a 181, localizada na mesorregião Agreste do Estado de Alagoas, nas coordenadas $9^{\circ} 45^{\prime} 09^{\prime \prime} \mathrm{S} \mathrm{e} 36^{\circ} 39^{\prime} 40^{\prime \prime} \mathrm{O}$, em altitude de $325 \mathrm{~m}$. Essa região é de transição entre a Zona da Mata e o Sertão alagoano, seu clima é do tipo 'As' tropical com estação seca de verão, pelo critério de classificação de Köppen (1948). De acordo com Xavier \& Dornellas (2010), a estação chuvosa tem início no mês de maio e se estende até a primeira quinzena de agosto, com precipitação média de $854 \mathrm{~mm}$ ano $^{-1}$, sendo os meses de maio a julho os mais chuvosos e os de setembro a dezembro os mais secos. O solo foi classificado como LATOSSOLO AMARELO VERMELHO Distrófico (Embrapa, 2013), com textura média a argilosa e densidade $\left(\mathrm{D}_{\mathrm{s}}\right)$ de $1,48 \mathrm{~g} \mathrm{~cm}^{-3}$.

Na Tabela 1,encontram-se as variáveis agrometeorológicas (temperatura, umidade relativa do ar, velocidade do vento e radiação solar) utilizadas para determinar a evapotranspiração de referência (ETo). A precipitação total no período foi de $202 \mathrm{~mm}$, com maior quantidade de chuva igual a 24,83 mm, no dia 08/05/2016.

Cinco lisímetros de drenagem foram utilizados, com espaçamento de 1,0 m entre eles, distribuídos em dois canteiros, cada um com as dimensões de 3,0 x 1,0 m de comprimento e largura, respectivamente. Os lisímetros foram construídos a partir de recipientes plásticos com as dimensões de 0,30 de diâmetro por 0,40 $\mathrm{m}$ de profundidade, respectivamente, que corresponde a uma área superficial de $0,07 \mathrm{~m}^{2}$.

$\mathrm{Na}$ área dos lisímetros, foi instalado um sistema de irrigação por gotejamento, utilizando-se gotejadores com vazão média de $1,0 \mathrm{~L} \mathrm{~h}^{-1}$. O solo foi colocado na capacidade de campo, objetivando o controle diário da lâmina e do tempo de irrigação. O volume irrigado diariamente variou em função do estádio fenológico da cultura. Inicialmente foi aplicado 0,5 L por lisímetro e, com o desenvolvimento da cultura, essa quantidade foi aumentando, até o volume diário máximo de $1,25 \mathrm{~L}$.

O fornecimento de água para os lisímetros ocorreu por ação da gravidade, a partir de reservatórios com capacidade de 20 litros, instalados sobre uma estrutura de madeira a 1,5 $\mathrm{m}$ do solo.

Os dados meteorológicos utilizados na análise comparativa dos modelos matemáticos de estimativa de evapotranspiração de referência (ETo), por meio dos métodos de Penman-Monteith, de Hargreaves-Samani, da Radiação Solar, de Blaney-Criddle e de Priestley-Taylor, foram obtidos de estação meteorológica do Instituto Nacional de Meteorologia (INMET), localizada a aproximadamente a $8,0 \mathrm{~km}$ da área experimental. 
A estimativa da evapotranspiração diária pelo método de Penman-Monteith foi estimada utilizando a Equação 1 (Allen et al., 1998):

$$
E t_{0}=\frac{0,408 \Delta(\mathrm{Rn}-\mathrm{G})+\gamma+\frac{900}{(\mathrm{~T}+273)} U 2(e a-e s)}{\Delta+\gamma+(1+0,34 \mathrm{U} 2)}
$$

em que: $E T o=$ evapotranspiração de referência $\left(\mathrm{mm} \mathrm{dia}^{-1}\right)$; $\mathrm{R}_{\mathrm{n}}=$ saldo de radiação diário $\left(\mathrm{MJ} \mathrm{m}^{-2} \operatorname{dia}^{-1}\right) ; \mathrm{G}=$ fluxo total diário de calor do solo $\left(\mathrm{MJ} \mathrm{m}^{-2} \mathrm{dia}^{-1}\right) ; \mathrm{T}=$ temperatura média diária do $\operatorname{ar}\left({ }^{\circ} \mathrm{C}\right) ; U 2=$ velocidade do vento média diária à altura de $2 \mathrm{~m}\left(\mathrm{~m} \mathrm{~s}^{-1}\right)$; es = pressão de saturação do vapor médio diário $(\mathrm{kPa})$; ea = pressão atual do vapor médio diário $(\mathrm{kPa}) ;($ es-ea $)=$ déficit de saturação de vapor médio diário $(\mathrm{kPa}) ; \Delta=$ declividade da curva da pressão do vapor em relação à temperatura $\left(\mathrm{kPa}^{\circ} \mathrm{C}^{-1}\right) ; \gamma=$ coeficiente psicrométrico $\left(\mathrm{kPa}^{\circ} \mathrm{C}^{-1}\right)$.

O método de Hargreaves-Samani, para estimativa da (ETo) diária, em $\mathrm{mm} \mathrm{d}^{-1}$, foi apresentado por Pereira et al. (1997):

$E T o=\alpha R a($ Tmed $+17,8)(\operatorname{Tmax}-\operatorname{Tmin})^{1 / 2}$

em que: $E T o=$ evapotranspiração de referência $\left(\mathrm{mm} \mathrm{dia}^{-1}\right)$; $\alpha=$ coeficiente adimensional, sendo: á $=0,0023 ; R a=$ Radiação extraterrestre $\left(\mathrm{mm} \mathrm{d}^{-1}\right)$; Tmed $=$ temperatura média diária $\left({ }^{\circ} \mathrm{C}\right) ;$ Tmax $=$ temperatura máxima $\left({ }^{\circ} \mathrm{C}\right)$ e Tmin $=$ temperatura mínima $\left({ }^{\circ} \mathrm{C}\right)$.

Para o método da Radiação Solar para a estimativa da ETo, utilizou-se a proposta apresentada por Doorenbos \& Pruitt (1977) que utiliza as Equações 3 e 4:

$E t_{0}=a+b W \frac{R s}{\lambda}$

$b=b 0+b 1$ URmed $+b 2 U+b 3$ URmed $U+b 4 U R 2+$ $+b 5 U 2$

em que: $E T o=$ evapotranspiração de referência $\left(\mathrm{mm} \mathrm{d}^{-1}\right) ; a$ $=$ coeficiente linear da reta $\left(a=-0,3 \mathrm{~mm} \mathrm{~d}^{-1}\right) ; b=$ coeficiente angular da reta $(b 0=1,0656 ; b 1=-0,0012795 ; b 2=0,044953$; $b 3=-0,00020033 ; b 4=-0,000031508 ; b 5=-0,0011026) ; W=$ índice de ponderação dependente da temperatura média, sendo: $W=0,407+0,0145$ Tmed $\left(\right.$ para $\left.0{ }^{\circ} \mathrm{C}<\mathrm{T}<16^{\circ} \mathrm{C}\right)$; $\mathrm{W}$
$=0,483+0,01 \mathrm{~T}\left(\right.$ para $\left.\mathrm{T} \mathrm{e} " 16^{\circ} \mathrm{C}\right) ; R s=$ radiação solar de ondas curtas recebida pela superfície terrestre em um plano horizontal, expressa em equivalente de evaporação (MJ $\left.\mathrm{m}^{-2} \mathrm{~d}^{-1}\right) ; \lambda=$ calor latente de evaporação $\left(\mathrm{MJ} \mathrm{kg}^{-1}\right)$, sendo: $\lambda$ H" 2,45; URmed = umidade relativa média (\%); $\mathrm{U}=$ velocidade média do vento à altura de $2 \mathrm{~m}\left(\mathrm{~m} \mathrm{~s}^{-2}\right)$; Tmed $=$ temperatura média $\left({ }^{\circ} \mathrm{C}\right)$.

No caso do método de Blaney-Criddle utilizou-se a versão mais conhecida que é a apresentada por Doorenbos \& Pruitt (1977) modificada por Frevert et al. (1983), de acordo com as Equações 5, 6, 7 e 8:

$$
\begin{aligned}
& \text { Eto }=a+b p(0,457 \text { Tmed }+8,13) \\
& \begin{array}{l}
A=a 0 U \text { Rmin }+a 1-\frac{n}{N} \\
b=b 0+b 1 \text { Urmin }+b 2 \frac{n}{N}+b 3 U 2+b 4 U \operatorname{Rmin} \frac{n}{N}+ \\
+b 5 U \text { Rmin U2 }
\end{array}
\end{aligned}
$$

em que: $E T o=$ evapotranspiração de referência $\left(\mathrm{mm} \mathrm{d}^{-1}\right)$; " $a$ " e " $b$ " = são coeficientes de ajuste (fator de correção), sendo: $a 0=0,0043$, a1 $=-1,41, b 0=0,81917, b 1=-0,0040922$, $b 2=1,0705, b 3=0,065649, b 4=-0,0059684$ e $b 5=-$ 0,$0005967 ; p=$ fator de correção função da latitude e época do ano, tabelado; Tmed $=$ Temperatura média do período $\left({ }^{\circ} \mathrm{C}\right) ;$ URmin = Umidade relativa mínima do período $(\%)$; $U 2=$ velocidade do vento média à altura de $2 \mathrm{~m}\left(\mathrm{~m} \mathrm{~s}^{-1}\right) ; n /$ $N=$ razão da insolação do período pelo fotoperíodo (horas), sendo: $n=$ insolação (h) que, por causa da ausência de medições em campo, considerou-se a equação de Angström-Prescott para sua estimativa; $N=$ fotoperíodo (h).

O método físico de Priestley-Taylor considera que a ETo proveniente do termo aerodinâmico, ou seja, do poder evaporante do ar, é uma percentagem da ETo condicionada pelo termo energético, baseado no método original de Penman. Assim, mesmo levando em consideração o balanço de energia, esse método apresenta um componente empírico. O método de Priestley-Taylor (1972) desenvolvido na Austrália, utilizando a variável saldo de radiação (Rn), é representado pela Equação 8.

$$
E t_{0}=\alpha\left(\frac{\Delta}{\Delta+\gamma}\right)\left(\frac{R n-G}{\lambda}\right)
$$

\begin{tabular}{|c|c|c|c|c|c|c|c|c|}
\hline & $\mathbf{T}_{\max }$ & $\mathbf{T}_{\text {med }}$ & $\mathbf{T}_{\min }$ & $\mathbf{U}_{\max }$ & $\mathbf{U}_{\text {med }}$ & $\mathbf{U}_{\min }$ & $\mathbf{V}$ & Rs \\
\hline & $\left({ }^{\circ} \mathrm{C}\right)$ & $\left({ }^{\circ} \mathrm{C}\right)$ & $\left({ }^{\circ} \mathrm{C}\right)$ & $(\%)$ & $(\%)$ & $(\%)$ & $\left(\mathrm{m} \mathrm{s}^{-1}\right)$ & $\left(\mathrm{MJ} \mathrm{m}^{-2}\right)$ \\
\hline Maior & 33,00 & 23,70 & 97,00 & 97,00 & 78,00 & 87,00 & 3,53 & 34,39 \\
\hline Média & 30,25 & 21,38 & 95,14 & 95,14 & 51,55 & 73,35 & 1,88 & 21,20 \\
\hline Menor & 26,30 & 18,80 & 92,00 & 92,00 & 34,00 & 64,00 & 0,88 & 11,38 \\
\hline
\end{tabular}

Tabela 1: Dados de temperatura máxima $\left(\mathrm{T}_{\max }\right)$, temperatura média $\left(\mathrm{T}_{\text {med }}\right)$, temperatura mínima $\left(\mathrm{T}_{\min }\right)$, umidade relativa do ar máxima $\left(\mathrm{U}_{\max }\right)$, umidade relativa do ar média $\left(\mathrm{U}_{\operatorname{med}}\right)$, umidade relativa do ar mínima $\left(\mathrm{U}_{\min }\right)$, velocidade do vento $(\mathrm{V})$ e radiação Solar (Rs) da unidade experimental do Grupo Irriga do Campus de Arapiraca, da UFAL, de março a julho de 2016

Fonte: INMET (2018) 
em que: $E T o=$ evapotranspiração de referência, $\left(\mathrm{mm} \mathrm{d}^{-1}\right)$; $\alpha=$ fator de ajuste universalmente conhecido como parâmetro de Priestley-Taylor, sendo: $\alpha=1,26$; $G=$ fluxo de calor do solo ( $\mathrm{MJ} \mathrm{m}^{-2} \mathrm{dia}^{-1}$ ), $\operatorname{com} G=0 ; \Delta=$ declividade da curva da pressão do vapor em relação à temperatura $\left(\mathrm{kPa}^{\circ} \mathrm{C}^{-1}\right) ; \gamma=$ coeficiente psicrométrico $\left(\mathrm{kPa}^{\circ} \mathrm{C}^{-1}\right) ; R n=$ saldo de radiação ( $\left.\mathrm{MJ} \mathrm{m}^{-2} \mathrm{dia}^{-1}\right) ; \lambda=$ calor latente de evaporação $\left(\mathrm{MJ} \mathrm{kg}^{-1}\right), \operatorname{com} \lambda=2,45$.

A aplicação da lâmina diária de irrigação nos lisímetros, a mensuração da água fornecida nos lisímetros e a coleta de água dos drenos para determinação da evapotranspiração da cultura (ETc) foi realizada com proveta volumétrica de $0,50 \mathrm{~L}$ a cada 24 horas, tendo início sempre às 13:00 h e término às 14:00 h.

Conforme a metodologia de Alves et al. (2017), o solo foi saturado durante 24 horas e em seguida colocado em capacidade de campo, observando-se a lâmina drenada, com o objetivo de determinar a primeira lâmina diária $(I)$, podendo-se aplicar as lâminas subsequentes, observando-se cuidadosamente o consumo diário $(L a-L d)$. Para as lâminas subsequentes, foram somadas ao consumo diário $50 \%$ deste, garantindo a drenagem diária e a lâmina aplicada diariamente. Para os cálculos, utilizaram-se as Equações 9 e 10, descritas por Alves et al. (2017).

$I=L a-L d+0,5(L a-L d)$

em que: $I=$ Irrigação diária aplicada (L); $L a=$ Lâmina aplicada diariamente nos lisímetros $(\mathrm{L}) ; L d=$ Lâmina drenada diariamente dos lisímetros (L).

$E T c=P+I-D$

em que: $E T c=$ evapotranspiração da cultura $(\mathrm{mm}) ; P=$ precipitação pluviométrica (mm); $I$ = lâmina de água aplicada por irrigação $(\mathrm{mm}) ; D=$ água drenada do lisímetro $(\mathrm{mm})$.

O coeficiente de cultura $(\mathrm{Kc})$ foi calculado pela razão entre a ETc e a ETo (Equação 11).

$K c=\frac{E t_{0}}{E t c}$

em que: $K c=$ coeficiente da cultura, adimensional; ETc $=$ evapotranspiração da cultura, $\mathrm{mm} \mathrm{d}^{-1} ;$ ETo $=$ evapotranspiração de referência, $\mathrm{mm} \mathrm{d}^{-1}$.

Após a coleta dos dados de ETc, obtidos dos lisímetros de drenagem, e de ETo pelas equações empíricas para cada método, foi determinado o Kc para cada fase fenológica da cultura da cenoura.

Para comparação e análise dos métodos de ETo’s foram utilizados critérios envolvendo o erro padrão de estimativa (EPE), erro padrão da estimativa ajustado (EPEa), coeficientes de ajustes das equações lineares e respectivos coeficientes de determinação $\left(\mathrm{R}^{2}\right)$ (Jensen et al. 1990).
As correlações entre os métodos de estimativas de ETo foram realizadas pelas análises de correlação e de regressão linear utilizando-se o programa estatístico R ( R Core Team, 2018) para obtenção dos coeficientes e do coeficiente de determinação $\left(\mathrm{R}^{2}\right)$.

Para a exatidão dos métodos empíricos foi determinado o índice de desempenho (c), calculado pelo produto do coeficiente de correlação (r) de Pearson e do índice de concordância (d) de Willmott et al. (1985). A correlação foi realizada entre os métodos de Hargreaves-Samani, da Radiação Solar, de Blaney-Criddle e de Priestley-Taylor, em relação ao método padrão de Penman-Monteith, a fim de se observar a precisão dada pelo coeficiente de correlação (r) que está associado ao desvio entre valores estimados e medidos, indicando o grau de dispersão dos dados obtidos em relação à média. Os valores de "r" e de "c" encontrados foram classificados conforme Willmott et al.(1985).

\section{RESULTADOS E DISCUSSÃO}

\section{Desempenho dos métodos de estimativa da ETo}

Na Figura 1 são apresentados os comportamentos da ETo estimados pelos métodos de Hargreaves-Samani,da Radiação Solar, de Blaney-Criddle, de Priestley-Taylor e de Penman-Monteith (método padrão) durante o ciclo de cultivo.

Analisando a Figura observou-se que a ETo estimada pelo método da Radiação Solar e de Blaney-Criddle subestimaram os valores de ETo, enquanto o método de Hargreaves-Samani subestimou esses valores em relação ao método padrão de Penman-Monteith. Os dados de ETo obtidos pelo método de Priestley-Taylor foram os que mais se aproximaram da ETo obtida pelo método padrão.

Na Figura 2, pode ser observada a correlação dos valores de ETo estimados pelos métodos de HargreavesSamani, da Radiação Solar, de Blaney-Criddle e de PriestleyTaylor com o método padrão de Penman-Monteith.

Analisando-se os coeficientes angulares das retas obtidas com as comparações dos métodos, pode ser observado que o coeficiente angular da equação da Figura 2A apresentou valor menor que 1 , indicando que os valores contidos no eixo X, método padrão de Penman-Monteith, são superiores aos encontrados no eixo Y,método de Hargreaves-Samani. Tal resultado evidenciou a subestimação dos valores pelo método de Hargreaves-Samani em relação aos do método padrão. Resultado semelhante foi obtido por Santos et al. (2016), onde a estimativa pelo método de Hargreaves-Samani foi menor do que pelo método de Penman-Monteith no Agreste Alagoano. Lima Junior et al. (2016), no Ceará, também constataram que o método de Hargreaves-Samani subestima a ETo, em relação à do método padrão. 


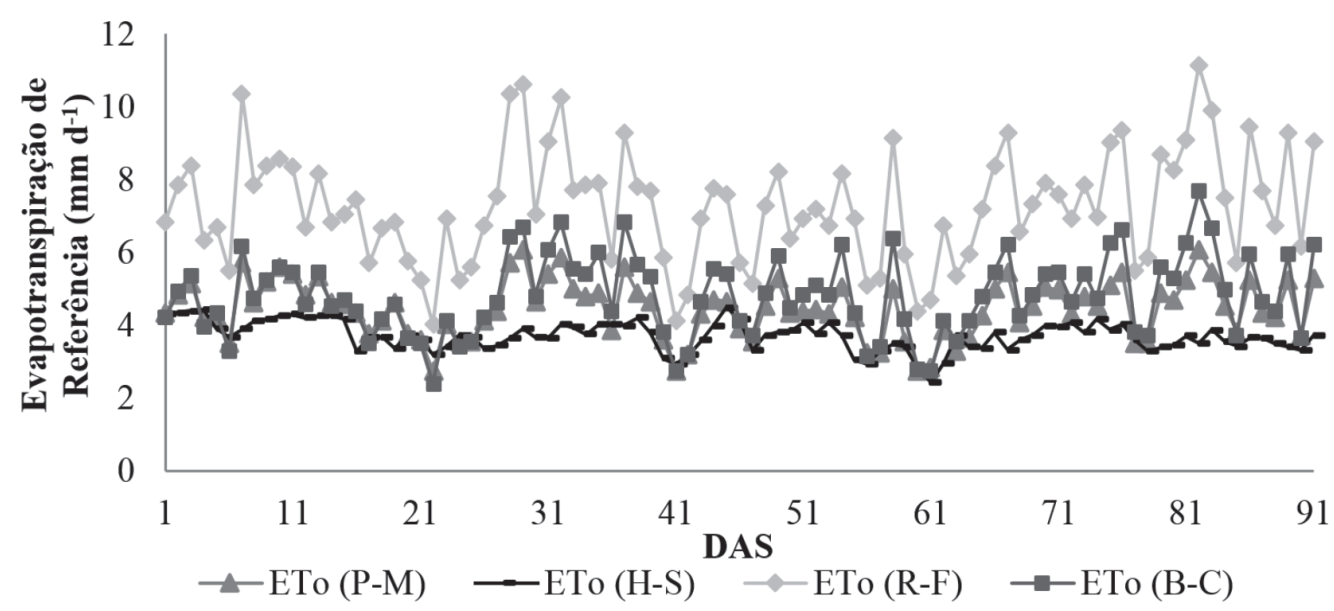

Figura 1: Evapotranspiração de referência (ETo) estimados pelos métodos de: Penman-Monteith $\left[\mathrm{ETo}_{(\mathrm{P}-\mathrm{M})}\right]$, Hargreaves-Samani $\left[\mathrm{ETo}_{(\mathrm{H}-\mathrm{S})}\right]$, Radiação Solar $\left[\mathrm{ETo}_{(\mathrm{R}-\mathrm{S})}\right]$, Blaney-Criddle $\left[\mathrm{ETo}_{(\mathrm{B}-\mathrm{C})}\right]$ e Priestley-Taylor $\left[\mathrm{ETo}_{(\mathrm{P}-\mathrm{T})}\right]$.
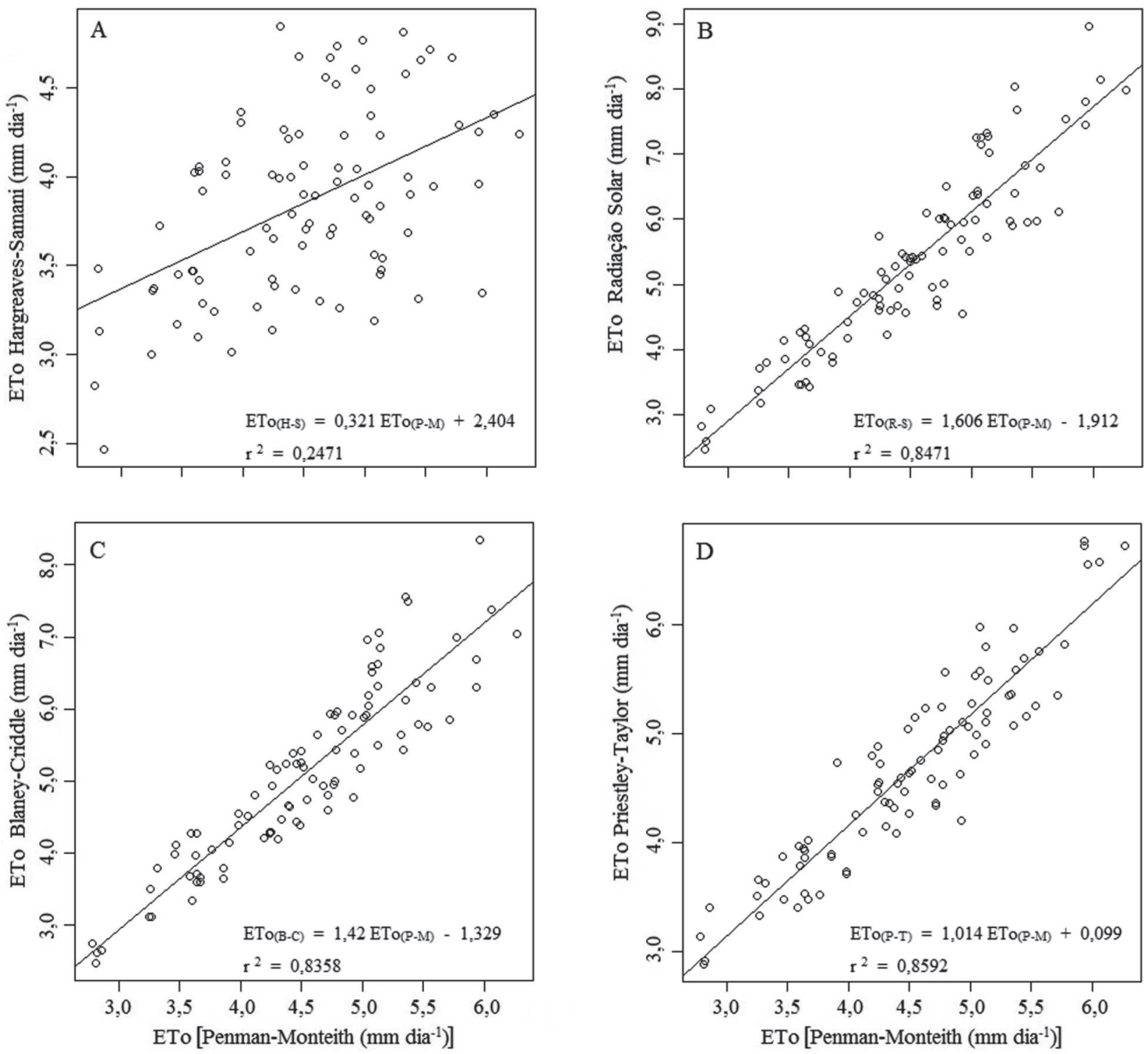

Figura 2: Correlação entre os valores diários da evapotranspiração de referência (ETo) $\left(\mathrm{mm}_{\text {.dia }}{ }^{-1}\right)$ estimados pelos métodos de Penman-Monteith [ETo $\left.{ }_{(\mathrm{P}-\mathrm{M})}\right]$, em relação aos métodos (A) de Hargreaves-Samani $\left[\mathrm{ETo}_{(\mathrm{H}-\mathrm{S})}\right]$, (B) da Radiação Solar $\left[\mathrm{ETo}_{(\mathrm{R}-\mathrm{S})}\right],(\mathrm{C})$ de Blaney-Criddle $\left[\mathrm{ETo}_{(\mathrm{B}-\mathrm{C})}\right]$ e (D) de Priestley-Taylor $\left[\mathrm{ETo}_{(\mathrm{P}-\mathrm{T})}\right]$. 
O coeficiente angular da equação da Figura 2B apresenta valor maior que 1 , indicando que os valores de ETo encontrados no eixo X, método de Penman-Monteith, são inferiores aos encontrados no eixo Y, método da Radiação Solar, evidenciando superestimação dos valores de ETo pelo método de Radiação Solar. O mesmo foi constatado para os dos valores de ETo pelo método de Blaney-Criddle (Figura 2C) e de Priestley-Taylor (Figura 2D) em relação ao método de Penman-Monteith.

Moura et al. (2013), em Vitória de Santo Antão, PE, encontrou uma superestimativa do método de BlaneyCriddle em relação ao método padrão. Tagliaferre et al. (2010), para as condições climáticas do Município de Eunápolis, BA, concluíram que o método de PriestleyTaylor subestimou os valores de evapotranspiração obtidos pelo método padrão de Penman-Monteith.

Na Tabela 2, observou-se que a ETo estimada pelo método de Hargreaves-Samani apresentou valores altos do EPE e do EPE $a$, com baixos valores dos coeficientes de correlação (r) e determinação $\left(\mathrm{r}^{2}\right)$, também apresentou desempenho sofrível $(\mathrm{d} ; \mathrm{c})$ de acordo com as estimativas dos valores de ETo obtidas neste estudo, indicando que o método não é preciso para estimar a ETo para a região de estudo. Este resultado pode ser explicado pela ocorrência de $202 \mathrm{~mm}$ de chuva, durante o período do experimento, condição favorável para influenciar nos parâmetros de ajustamento da do método de Hargreaves-Samani, uma vez que este método é recomendado para regiões áridas. $\mathrm{O}$ modelo de Hargreaves-Samani tende a superestimar a ETo em regiões úmidas, como observado por diversos autores (Alencar et al., 2011; Alencar et al., 2015; Tanaka et al., 2016).

Dentre os modelos estudados, o método de HargreavesSamani é o método mais dependente e diretamente relacionado com a temperatura do ar. Segundo Lyra et al. (2011), a temperatura do ar é influenciada diretamente pela radiação; entretanto, outros fatores podem também influenciála, como a umidade do ar. Lima Junior et al. (2016) constataram que, em praticamente todas as localidades estudadas por eles no Ceará, em regiões de litoral e nas semiáridas, que a ETo estima por Hargreaves-Samani superestimou a ETo estimada por Penman-Monteith nos primeiros meses do ano, período esse que apresenta maiores quantidades de chuvas e menores amplitudes térmicas.

O baixo desempenho do método de Hargreaves-Samani também foi constatado por Lucena et al. (2016) no município de Bom Jesus, PI., quando compararam a ETo nos períodos chuvoso e seco. O método apresentou desempenho sofrível $(\mathrm{d}=0,99 ; \mathrm{c}=0,57)$, para o método de Hargreaves-Samani no período de chuvas, enquanto, no período seco, o método de Hargreaves-Samani apresentou-se com desempenho caracterizado como bom, com índices $(d=0,99 ; c=0,72)$.

Para o método da Radiação Solarforam encontrados altos valores de coeficientes de correlação (r) e de determinação $\left(\mathrm{r}^{2}\right)$, sendo o método classificado como de desempenho bom. Porém, quando analisados os erros, EPE e EPE $a$, foram encontrados valores elevados, o que indica que o método não é adequado para a região e período de estudo (Tabela 2). Esses resultados corroboram os de Chagas et al. (2013) que trabalhou no município de Rio Real, Bahia, e os de Mendonça \& Dantas (2010), em estudo realizado em Capim, PB.

Para o método de Blaney-Criddle foram encontrados altos valores dos coeficientes de correlação (r) e de determinação $\left(\mathrm{r}^{2}\right)$, sendo o método classificado como de desempenho muito bom para os valores de "d" e de "c" (Tabela 2). Para o método de Blaney-Criddle, tanto Almeida et al. (2010), em Fortaleza, CE, quanto Cavalcante Junior et al. (2010), em Mossoró, RN, obtiveram desempenho "ótimo" e excelente $\mathrm{r}^{2}$. Porém, quando analisados os erros para o método de Blaney-Criddle foram encontrados valores altos de erros EPE e EPE $a$, indicando que o método não se adequa à região e ao período de estudo (Tabela 2).

Ainda na Tabela 2, observou-se que os maiores valores de coeficientes de correlação ( $\mathrm{r})$ e de determinação $\left(\mathrm{r}^{2}\right)$ foram obtidos pelo método de Priestley-Taylor. Os valores dos coeficientes estatísticos apresentaram superestimativa dos valores de ETo para o método de Priestley-Taylor em relação aos do método de Penman-Monteith e houve razoável precisão (r), assim como a exatidão (d). De acordo com os valores de EPE e EPE $a$, observou-se que os erros para o método de Priestley-Taylor são os menores encontrados neste trabalho, o que demonstra ser esse o método que mais se aproxima do método de Penman-Monteith.

Tabela 2: Erro padrão de estimativa (EPE) e da estimativa ajustado (EPEa), coeficientes de correlação $(r)$ e de determinação $\left(r^{2}\right)$ e índices de concordância $(d)$ e de desempenho entre os métodos de Hargreaves-Samani $\left[\mathrm{ETo}_{(\mathrm{H}-\mathrm{S})}\right]$, Radiação Solar $\left[\mathrm{ETo}_{(\mathrm{R}-\mathrm{S})}\right]$, BlaneyCriddle $\left[\mathrm{ETo}_{(\mathrm{B}-\mathrm{C})}\right]$ e Priestley-Taylor $\left[\mathrm{ETo}_{(\mathrm{P}-\mathrm{T})}\right]$ e o método padrão de Penman-Monteith $\left[\mathrm{ETo}_{(\mathrm{P}-\mathrm{M})}\right]$

\begin{tabular}{|c|c|c|c|c|c|c|}
\hline \multirow{2}{*}{ ETo $_{(\mathrm{P}-\mathrm{M})} \mathbf{x}$... } & \multirow{2}{*}{ EPE } & \multirow{2}{*}{ EPEa } & $r$ & $r^{2}$ & $d$ & $c$ \\
\hline & & & \multicolumn{4}{|c|}{$(\%)$} \\
\hline $\mathbf{E T o}_{(\mathrm{H}-\mathrm{S})}$ & 0,90 & 0,86 & 49,71 & 24,71 & 46,70 & 23,21 \\
\hline $\mathbf{E T o}_{(\mathrm{R}-\mathrm{S})}$ & 1,10 & 0,96 & 92,04 & 84,71 & 71,82 & 66,10 \\
\hline $\mathbf{E T o}_{(\mathrm{B}-\mathrm{C})}$ & 0,83 & 0,66 & 91,42 & 83,58 & 83,96 & 76,76 \\
\hline $\mathbf{E T o}_{(\mathrm{P}-\mathrm{T})}$ & 0,37 & 0,16 & 92,69 & 85,92 & 95,05 & 88,11 \\
\hline
\end{tabular}




\section{ETc e Kc da cultura da cenoura}

Os valores referentes à evapotranspiração da cultura (ETc) da cenoura, obtidos por meio da lisimetria de drenagem são representados na Figura 3.Inicialmente foram verificadas pequenas variações na demanda de água pela cultura. A partir dos 30 DAS, o consumo foi aumentando gradativamente, atingindo um nível máximo de $9,56 \mathrm{~mm}$, aos 71 DAS, nas fases de crescimento vegetativo e de desenvolvimento da raiz. O consumo hídrico obtido pelos lisímetros de drenagem para a cultura da cenoura, em um ciclo de 91 dias, foi de 421,00 mm. Resultados diferentes dos encontrados por Santos et al. (2009), registraram um consumo médio de $811,84 \mathrm{~mm}$ para a cultura da cenoura, no Agreste de Pernambuco, utilizando lisímetro de drenagem.

Essas diferenças devem-se principalmente ao fato de os cultivos não serem realizados no mesmo período. $\mathrm{O}$ estudo de Santos et al. (2009) foi realizado na estação seca, quando ocorre maior demanda hídrica, enquanto este estudo foi realizado na estação chuvosa.

$\mathrm{Na}$ Tabela 3,encontram-se os valores de Kc ao longo do ciclo fenológico da cultura, obtidos pelas metodologias de estimativa da ETo de: PenmanMonteith, Hargreaves-Samani, Radiação Solar, BlaneyCriddle e Priestley-Taylor.

Os valores de Kc obtidos na fase inicial foram semelhantes para todos os métodos 0,$46 ; 0,49 ; 0,44 ; 0,46$ e 0,46 , respectivamente para os métodos de Penman-Monteith (FAO-56), de Hargreaves-Samani, da Radiação Solar, de Blaney-Criddle e de Priestley-Taylor, sendo distintos nas demais fases (Tabela 3 ).

Como a planta transpira menos no início do seu desenvolvimento, $\mathrm{o} \mathrm{Kc}_{\text {incial }}$ é dominado pelos fatores ambientais, quando predomina a evaporação da água no solo, o que resultou em valores maiores de ETo e menores ETc, fazendo que o Kc inicialmente seja menor do que em outras fases.

A partir da fase fenológica de desenvolvimento vegetativo, passando para o desenvolvimento da raiz, os valores de Kc elevaram-se (Tabela 3). Essas fases são caracterizadas por fotossíntese intensa, necessária para o crescimento dos tecidos e o armazenamento de carboidratos nos tecidos de reserva. Já na fase de maturação, o Kc decresceu, pois a planta encontra-se em desenvolvimento pleno e não está mais armazenando energia, além de apresentar decréscimo da área foliar, o que resulta em menor consumo de água pela planta. Houve variação dos valores de Kc para cada fase fenológica, em função do método avaliado. O método de Hargreaves-Samani superestimou, em relação a Penman-Monteith, os valores de Kc, enquanto Radiação solar subestimou os valores em relação a Penman-Monteith, ambos em todas as fases de desenvolvimento. Blaney-Criddle também subestimou os valores de Kc em relação aos de Penman-Monteith, nas fases II, III

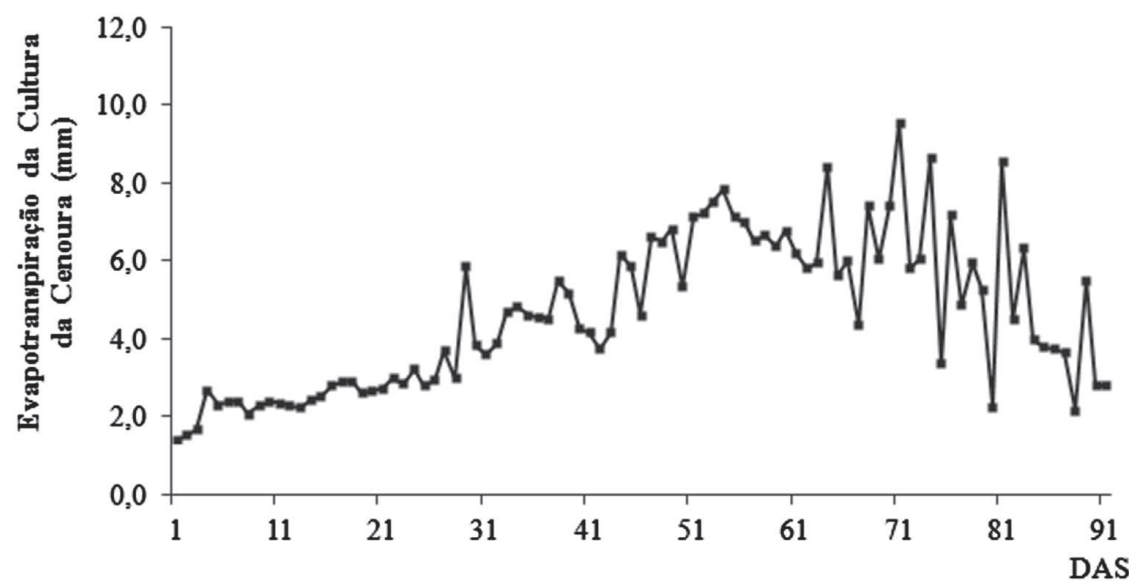

Figura 3: Evapotranspiração da cultura (ETc) no período de realização do experimento para as condições do agreste alagoano.

Tabela 3: Coeficientes da cultura $(\mathrm{Kc})$ da cenoura em diferentes períodos e fases de desenvolvimento pelos métodos de PenmanMonteith (P-M), Hargreaves-Samani (H-S), Radiação Solar (R-S), Blaney-Criddle (B-C) e Priestley-Taylor (P-T)

\begin{tabular}{lccccccc}
\hline \multirow{2}{*}{ Período } & \multicolumn{1}{c}{ Kc } & P-M & H-S & R-S & B-C & P-T \\
\cline { 2 - 8 } & Fases & dias & P-M & 0,46 \\
\hline Semeadura à germinação & I & 30 & 0,46 & 0,49 & 0,44 & 0,46 & $0,46 \sim 1,26$ \\
Desenvolvimento da cultura & II & 15 & $0,46 \sim 1,31$ & $0,49 \sim 1,49$ & $0,44 \sim 1,12$ & $0,46 \sim 1,16$ & $0,46 \sim 1,26$ \\
Formação das raízes & III & 35 & 1,31 & 1,49 & 1,12 & 1,16 & 1,26 \\
Maturação & IV & 10 & $1,31 \sim 1,09$ & $1,49 \sim 1,26$ & $1,12 \sim 0,95$ & $1,16 \sim 0,99$ & $1,26 \sim 1,05$ \\
\hline
\end{tabular}


e IV, mas não na fase I, em que o componente ambiental tem maior influência.

Evidenciou-se que valores superestimados de ETo resultaram em valores subestimados de Kc, enquanto valores subestimados de ETo resultam em valores superestimados de Kc.

Oliveira et al. (2003), trabalhando com cenoura cultivada na região do Alto Parnaíba, MG, com Kc basal ajustado com as condições de clima, encontraram valores de Kc de 1,15 , na fase inicial, 1,12 , na fase de crescimento, 1,12 , na fase intermediária e 1,10, na fase final. A região estudada por Oliveira et al. (2003) difere bastante das condições da área de estudo deste trabalho e a análise dos resultados indicou que a maior diferença encontrada entre os Kc's, comparados fase por fase, deu-se principalmente nas fases I e IV, já que, nessas fases o valor de Kc é dominado pelos fatores ambientais.

\section{CONCLUSÕES}

O método de Priestley-Taylor apresentou o melhor desempenho na estimativa da evapotranspiração de referência, entre os métodos estudados, em relação ao de PenmanMonteith, sendo recomendado para determinação da ETo para a região.

A demanda hídrica diária da cultura da cenoura, em período chuvoso, para região agreste alagoana, foi de 4,62 $\mathrm{mmd}^{-1}$.

O Kc da cenoura, recomendado para a região, é 0,46 ; 1,3; 1,3 e 1,09, para as fases I, II, III e IV respectivamente, obtidos pelo método padrão.

$\mathrm{Na}$ ausência de informações agrometeorológicas para se estimar a ETo pelo método padrão, recomendam-se os valores de Kc de 0,46; 1,26; 1,26 e 1,05, obtidos pelo método de ETo de Priestley-Taylor.

\section{REFERÊNCIAS}

Allen RG, Pereira SL, Raes D \& Smith M (1998) Crop evapotranspiration - Guidelines for computing crop water requirements. Rome, FAO. 15p. (Irrigation and Drainage, 56).

Alencar LP, Delgado RC, Almeida TS \& Wanderley HS (2011) Comparação de diferentes métodos de estimativa diária da evapotranspiração de referência para a região de Uberaba. $\mathrm{Re}$ vista Brasileira de Ciências Agrárias, 6:337-343.

Alencar LP, Sediyama GC \& Mantovani EC (2015) Estimativa da evapotranspiração (ET0 padrão FAO), para Minas Gerais, na ausência de alguns dados climáticos. Revista Engenharia Agrícola, 35:39-50.

Almeida BM, Araújo EM, Cavalcante Júnior EG, Oliveira JB, Araújo EM \& Nogueira BRC (2010) Comparação de métodos de estimativa da ETo na escala mensal em Fortaleza-CE. Revista Brasileira de Agricultura Irrigada, 4:93-98.

Alves ES, Lima DF, Barreto JAS, Santos DP \& Santos MAL (2017) Determinação do coeficiente de cultivo para a cultura do rabanete através de lisimetria de drenagem. Revista Irriga, 22:194-203.
Araujo GL, Reis EF, Martins CAS, Barbosa VS \& Rodrigues RR (2011) Desempenho comparativo de métodos para a estimativa da evapotranspiração de referência (ETo). Revista Brasileira de Agricultura Irrigada, 5:84-95.

Carvalho ADF, Silva GO \& Resende FV (2017) Adaptabilidade e estabilidade de populações de cenoura pelo método REML/ BLUP. Horticultura Brasileira, 35:69-74.

Cavalcante Junior EG, Almeida BM, Oliveira AD, Espinola Sobrinho J, Araújo EM \& Vieira RY (2010) Estimativa da evapotranspiração de referência para a cidade de Mossoró-RN. Revista Brasileira de Agricultura Irrigada, 4:87-92.

Chagas RM, Faccioli GG, Aguiar Netto AO, Sousa IF, Vasco NA \& Silva MG (2013) Comparação entre métodos de estimativa da evapotranspiração de referência (ETo) no município de rio RealBA. Irriga, 18:351-363.

Doorenbos J \& Pruitt JO (1977) Guidelines for predicting crop water requeriments. Rome, FAO lrrigation and Drainage. 179p.

Embrapa - Empresa Brasileira de Pesquisa Agropecuária (2013) Sistema brasileiro de classificação de solos. Centro Nacional de Pesquisas de Solos. Rio de Janeiro, Embrapa Solos. 353p.

Frevert DR, Hill RW \& Braaten BC (1983) Estimation of FAO evapotranspiration coeficients. Journal of Irrigation and Drainage, 109:265-270.

INMET - Instituto Nacional de Meteorologia (2018) Disponível em: http://www.inmet.gov.br/portal/. Acessado em: 20 de fevereiro de 2017.

Jensen ME, Burman RD \& Allen RG (1990) Evapotranspiration and Irrigation Water Requirements. New York, The American Society of Civil Engineers. 332p.

Lima Junior JC, Arraes FDD, Oliveira JB, Nascimento FAL \& Macêdo KG (2016) Parametrização da equação de Hargreaves e Samani para estimativa da evapotranspiração de referência no Estado do Ceará, Brasil. Revista Ciência Agronômica, 47:447-454.

Lucena FAP, Silva EM, Ribeiro AA, Simeão M \& Lucena JPAP (2016) Comparação entre métodos de estimativa da evapotranspiração de referência no município de Bom Jesus, PI. Revista Brasileira de Agricultura Irrigada, 10:663-675.

Lyra GB, Santos MJ, Souza JL, Lyra GB \& Santos MA (2011) Espacialização da temperatura do ar anual para o estado de Alagoas com diferentes modelos digitais de elevação e resoluções espaciais. Ciência Florestal, 21:275-287.

Köppen W (1948) Climatologia: conune studio de los climas de latierra. México, Fondo de Cultura Economica. 478p.

Mendonça EA \& Dantas R (2010) Estimativa da evapotranspiração de referência no município de Capim, PB. Revista Brasileira de Engenharia Agrícola e Ambiental, 14:196-202.

Miranda LM, Carvalho LG, Neto PC \& Santos PAB (2016) Utilização do lisímetro de drenagem para obtenção do Kc da mamoneira em plantio adensado. Revista Verde de agroecologia e desenvolvimento sustentável, 11:08-13.

Moura ARC, Montenegro SGL, Antonino ACD, Azevedo JRG, Silva BB \& Oliveira M (2013) Evapotranspiração de referência baseada em métodos empíricos em Bacia experimental no estado de Pernambuco - Brasil. Revista Brasileira de Meteorologia, 28:181-191

Oliveira RA, Rocha IB, Sediyama GC, Puiatti M, Cecon PR \& Silveira SFR (2003) Coeficientes de cultura da cenoura nas condições edafoclimáticas do Alto Paranaíba, Minas Gerais. Revista Brasileira de Engenharia Agrícola e Ambiental, 7:280-284.

Oliveira VMR, Dantas GF, Palaretti LF, Dalri AB, Santos MG \& Ficher Filho JA (2015) Estimativa de evapotranspiração de referência na região de rio Paranaíba-MG. Irriga, 20:790-798. 
Pereira AR, Manieiro MA, Villa Nova NA \& Se GC (1997) Evapo(transpi)ração. Piracicaba, FEALQ. 183p.

Priestley CHB \& Taylor RJ (1972) On the assessment of surfasse heat flux and evaporation using large-scale parameters. Monthly Weather Review, 100:81-92.

R Core Team (2018) A language and environment for statistical computing. R Foundation for Statistical Computing. Disponível em: http://www.R-project.org/. Acessado em: 23 de dezembro de 2017.

Santos CS, Santos DP, Oliveira WJ, Silva PF, Santos MAL \& Fontenele AJPB (2016) Evapotranspiração de referência e coeficiente de cultivo da pimenteira no Agreste Alagoano. Revista Brasileira de Agricultura Irrigada, 10:883-892.

Santos FX, Montenegro AAA, Silva JR \& Souza ER (2009) Determinação do consumo hídrico da cenoura utilizando lisímetros de drenagem, no agreste pernambucano. Revista Brasileira de Ciências Agrárias, 4:304-310.
Silva TGF, Moura MSB, Zolnier S, Soares JM, Vieira VJS \& Júnior WGF (2012) Requerimento hídrico e coeficiente de cultura da cana-de-açúcar irrigada no semiárido brasileiro. Revista Brasileira de Engenharia Agrícola e Ambiental, 16:64-71.

Tanaka AA, Souza AP, Klar AE, Silva AC \& Gomes AWA (2016) Evapotranspiração de referência estimada por modelos simplificados para o Estado do Mato Grosso. Pesquisa Agropecuária Brasileira, 51:91-104.

Tagliaferre C, Jesus Silva RA, Rocha FA, Costa Santos L \& Silva CDS (2010) Estudo comparativo de diferentes metodologias para determinação da evapotranspiração de referência em Eunápolis-BA. Revista Caatinga, 23:103-111.

Xavier RA \& Dornellas PC (2010) Análise do comportamento das chuvas no município de Arapiraca, região Agreste de Alagoas. Geografia, 14:49-64.

Willmott CJ, Ackleson SG, Davis RE, Feddema JJ, Klink KM, Legates DR, Rowe CM \& O'Donnell J (1985) Statistics for the evaluation and comparison of models. Journal of Geophysical Research, 90:8995-9005. 